\title{
Is there a need for shifting patients on long term nevirapine based regimens to efavirenz based regimens: a cross-sectional study?
}

\author{
Nitin Gupta, Ankit Mittal, Kutty Sharada Vinod, Farhan Fazal, Wasim Khot, Sanjay Ranjan, \\ Neeraj Nischal*, Manish Soneja, Ashutosh Biswas, Naveet Wig, Rita Sood \\ Department of Medicine, All India Institute of Medical Sciences, New Delhi, India.
}

\begin{abstract}
Summary Non-nucleoside reverse transcriptase inhibitors (NNRTIs) are the backbone of effective anti-retroviral therapy in the developing world. Efavirenz is the current NNRTI of choice due to reports of higher incidence of serious adverse events with nevirapine. Majority of patients with Human immunodeficiency virus (HIV) infection in India are still on nevirapine based therapy. The aim of the study was to evaluate the need of shifting these patients to efavirenz based therapy. A cross-sectional study was conducted on adult patients, who were on NNRTI based regimen for more than one year with good adherence. The patients were divided into efavirenz or nevirapine groups based on the treatments they were receiving at the time of study. The different arms were compared based on their clinical and laboratory profile, adverse events and immunological response. A total of 244 patients were recruited. A total of 125 patients were receiving nevirapine based regimen while 119 patients were receiving efavirenz based regimen. There was no significant difference in the frequency of hematological and biochemical derangements between the two groups. There was no difference in the median highest $\mathrm{CD} 4$ count achieved during therapy between the two groups. Clinically observed side effects were more common in the efavirenz group. These results suggest that there isn't enough evidence to shift patients tolerating long term nevirapine based therapy to efavirenz based therapy.
\end{abstract}

Keywords: Adverse events, efficacy, non-nucleoside reverse transcriptase inhibitor

\section{Introduction}

Non-nucleoside reverse transcriptase inhibitors (NNRTI) form the backbone of the three-drug first line regimen against human immunodeficiency virus (HIV) infection in most parts of the world (1). It has been effectively used in the developing world for a long time owing to their low cost and high potency. In the World Health Organization (WHO) guidelines (antiretroviral therapy for HIV infection in adults and adolescents) published in 2010, both nevirapine and efavirenz were mentioned as preferred NNRTIs for the first line therapy (2). However, nevirapine slowly fell out of favor because of higher incidence of serious adverse

\footnotetext{
*Address correspondence to:

Dr. Neeraj Nischal, Department of Medicine, Teaching block, 3rd floor, All India Institute of Medical Sciences, New Delhi-110029, India.

E-mail: neerajnischal@gmail.com
}

events in early part of therapy, especially in individuals with higher CD4 counts (3). Therefore, in the WHO consolidated guidelines (Use of antiretroviral drugs for treating and preventing HIV infections) of 2013, efavirenz containing regimens were preferred as the first line therapy while nevirapine based regimens were moved to the alternative first line list (4). However, the question of shifting those patients, who were already on long term nevirapine based therapy to efavirenz containing regimens was still unanswered. The primary objective of this study was to therefore, compare the long-term tolerability of patients receiving nevirapine based regimen vs those receiving efavirenz based regimens.

\section{Methods}

A cross-sectional study was conducted after taking approval from the Institute's Ethics committee. Adult patients ( $>18$ years) on NNRTI based regimens for 
more than one year with good adherence ( $>95 \%$ adherence over last one year by pill count method) attending the anti-retroviral therapy (ART) center were recruited after taking informed consent.

A one-time interview was done based on a structured questionnaire. The patients were divided into efavirenz or nevirapine groups based on the NNRTI they were receiving. Patients were interviewed and examined for any adverse effects (clinician and patient reported) to ART. The following laboratory parameters were collected: complete blood count, liver/kidney function tests, and electrolyte levels. Their records were reviewed for treatment details and immunological parameters. CD4 count at baseline, highest CD4 count achieved during treatment and the most recent CD4 count were recorded. The highest change from the baseline was calculated by subtracting baseline CD4 count from the highest CD4 count. Immunological failure was defined as persistently low CD4 count $(<$ $100 / \mu \mathrm{L}$ )/decrease in CD4 count below the base line or half of the highest CD4 attained during the treatment (4). The two arms were compared based on their clinical and laboratory profile, clinical adverse events and immunological response.

Statistical analysis Data was collected on a predesigned pro forma. All data was presented as mean \pm SD or median and interquartile range. Frequency of each of the outcomes were expressed in percentage with 95\% confidence interval (CI) determined for each of the percentage. Appropriate parametric/non-parametric tests were used based on the type of variables and their distribution.

\section{Results and Discussion}

A total of 244 patients were recruited in the study, $68 \%$ (165) of whom were male. A total of 125 (51\%) patients were receiving nevirapine based regimen while 119 (49\%) were receiving efavirenz based regimen. Out of the 125 patients receiving nevirapine, zidovudine and lamivudine formed the rest of the regimen in 117 (94\%) patients. Out of the 119 patients receiving efavirenz, $108(91 \%)$ patients were also receiving tenofovir plus lamivudine. The patients were also classified into the following groups based on the year of initiation (20052008-40, 2009-2012-63, 2013-2017-141). Median baseline CD4 count at the time of initiation of treatment was $214.5 / \mu \mathrm{L}$ (121-320.5). Median highest CD4 count achieved during treatment was $591.5 / \mu \mathrm{L}$ (413.5-789.5). Median highest increase in CD4 count from the baseline was $362.5 / \mu \mathrm{L}$ (207.25-546.75). Immunological failure was noted in 12 patients. A total of 19 patients had a history of concurrent tuberculosis with HIV. A total of $33(13.5 \%)$ patients reported some clinical side effect during the interview. None of them were severe enough to require discontinuation. The following clinical side effects were reported by the patients: neuropsychiatric symptoms $(n=13)$, gastro-intestinal symptoms $(n=8)$, lipodystrophy $(n=5)$, peripheral neuropathy $(n=3)$, rash $(n=2)$ and gynecomastia $(n=2)$.

The following hematological abnormalities were noted: anemia $(<11 \mathrm{~g} / \mathrm{dL})-41$, leucopenia $(<$ $4,000 / \mathrm{mm} 3)-13$, leukocytosis $(>11,000 / \mathrm{mm} 3)$ 7 , thrombocytopenia $(<1,000,000 / \mathrm{mm} 3)-34$, and macrocytosis ( $>100 \mathrm{fl})-132$. The following biochemical abnormalities were noted: transaminitis-69, deranged kidney function-4, hypophosphatemia $(<2.5 \mathrm{mg} / \mathrm{dL})-53$ and hypocalcaemia $(<8.8 \mathrm{mg} / \mathrm{dL})-74$.

There was no significant difference between the nevirapine and efavirenz group in the following demographic features: locality, distance of home from the ART center, literacy, employment status, addictions and baseline CD4 count (Table 1). However, there was significant difference between the two groups in terms of mean age at the time of analysis and year of initiation. There was no significant difference in the frequency of hematological and biochemical derangements except for that of macrocytosis which was more common in the nevirapine group. There was no difference in the median highest CD4 count achieved during therapy but the median of highest change in CD4 count was significantly higher in the nevirapine group. Clinically observed side effects were more common with efavirenz (Table 1).

The treatment options for AIDS has drastically changed since 1987, when the first drug, zidovudine was approved (5). Although zidovudine was efficacious, it had serious side effects like bone marrow suppression. Also, monotherapy was eventually leading to resistance and failure. Since then, the world has moved on to effective combination therapy, commonly consisting of three drugs (6). Two of these drugs are Nucleoside Reverse Transcriptase Inhibitor (NRTI), while the third drug can be either an Integrase inhibitor (INSTI) or Protease inhibitor (PI) or Non-nucleoside reverse transcriptase inhibitor (6). The Department of Human and Health Services (DHHS) favors an integrase inhibitor based regimen because of its high barrier to resistance, high potency and less side effects (7). However, WHO and national guidelines in India still recommend a NNRTI based regimen owing to their lower cost. Although, NNRTIs have been associated with some side effects (Nevirapine- severe hypersensitivity, Efavirenz- central nervous system (CNS) side effects), most of them are observed in the initial few weeks to months after the initiation of treatment $(8)$. Both the drugs have shown to have lower frequency of side effects in patients who have been on these drugs for long durations. In our study, a total of only $13.5 \%$ patients had clinically observed side effects at the time of interview. This included side effects related to both NRTIs and NNRTIs. None of the side effects were severe enough to require discontinuation. However, clinical observed side effects, predominantly persistent neuropsychiatric symptoms were observed 
Table 1. Comparison of demographic characteristics, clinical and laboratory parameters between patients on nevirapine vs efavirenz based regimens

\begin{tabular}{|c|c|c|c|}
\hline Characteristics & Nevirapine $(n=125)$ & Efavirenz $(n=119)$ & $p$ value \\
\hline Age & $37.9 \pm 10$ & $35.3 \pm 9.1$ & 0.03 \\
\hline Year of initiation & & & $<0.01$ \\
\hline 2005-08 & 32 & 8 & \\
\hline $2009-12$ & 48 & 15 & \\
\hline 2013-16 & 45 & 96 & $<0.01$ \\
\hline Mean duration of therapy in years & 6.2 & 3.1 & 0.6 \\
\hline \multicolumn{4}{|l|}{ Locality } \\
\hline Urban & 110 & 107 & 0.8 \\
\hline Rural & 15 & 112 & \\
\hline Distance & & & 0.1 \\
\hline$<100 \mathrm{~km}$ & 97 & 94 & \\
\hline$>100 \mathrm{~km}$ & 28 & 25 & \\
\hline \multicolumn{4}{|l|}{ Literacy } \\
\hline Primary & 43 & 38 & \\
\hline Secondary & 49 & 36 & \\
\hline College & 17 & 16 & \\
\hline Illiterate & 16 & 29 & \\
\hline Employed & 83 & 75 & 0.6 \\
\hline Addiction (smoker or alcoholic) & 24 & 18 & 0.4 \\
\hline CD4 baseline & $192(102-310)$ & $232(157.5-324.5)$ & 0.2 \\
\hline Highest CD4 & $645(427-852)$ & $568(406-735)$ & 0.3 \\
\hline Change in CD4 & $423(237-600)$ & $288(199.5-447)$ & 0.005 \\
\hline Immunological failure & 6 & 6 & 0.6 \\
\hline Clinically observed side effects & & & 0.03 \\
\hline Overall (33) & 11 & 22 & \\
\hline Gastro-intestinal symptoms $(n=8)$ & 3 & 5 & \\
\hline Neuropsychiatric symptoms $(n=13)$ & 1 & 12 & \\
\hline Peripheral neuropathy $(n=3)$ & 1 & 2 & \\
\hline $\operatorname{Rash}(n=2)$ & 1 & 1 & \\
\hline Lipodystrophy $(n=5)$ & 5 & 0 & \\
\hline Gynaecomastia $(n=2)$ & 0 & 2 & \\
\hline Macrocytosis & 105 & 27 & $<0.01$ \\
\hline Anaemia & 17 & 24 & 0.2 \\
\hline Leucocytosis & 2 & 5 & 0.2 \\
\hline Leucopenia & 8 & 5 & 0.4 \\
\hline Thrombocytopenia & 18 & 16 & 0.8 \\
\hline Transaminitis & 36 & 33 & 0.8 \\
\hline Deranged KFT & 1 & 3 & 0.3 \\
\hline Hypocalcemia & 36 & 38 & 0.6 \\
\hline Hypophosphatemia & 32 & 32 & 0.1 \\
\hline
\end{tabular}

$" p<0.05 ; " p<0.01$ compared with control group.

more in the efavirenz group.

No significant difference in hematological and biochemical derangements were observed between the two groups except for that of higher frequency of macrocytosis in the nevirapine group. This was because nevirapine was co-administered with zidovudine in $94 \%$ of cases, which competes with deoxy nucleoside triphosphates and results in impaired synthesis of the erythrocyte precursor in the bone marrow (9).

In our study, although we did not find any difference between the highest CD4 count attained during treatment between the two groups, there was a higher median CD4 count change (between baseline and highest) in the nevirapine group. This was probably due to the longer duration of treatment in the nevirapine group. In a meta-analysis by Cochrane infectious diseases group, no significant difference was observed between nevirapine and efavirenz in terms of virological success and progression to AIDS (10). Studies from India have shown good tolerability with both nevirapine and efavirenz (11-14).

In conclusion, since most serious adverse events associated with nevirapine are seen during the first few weeks/months of the therapy, nevirapine might be as good as efavirenz, if not better, in those patients who are tolerating it well for years. There isn't enough evidence for shifting the patients on long term nevirapine based therapy to efavirenz containing regimen.

Limitations of the study: This was a cross-sectional study without any follow up and the data was collected from a single interview.

\section{References}

1. Sashindran VK, Chauhan R. Antiretroviral therapy: Shifting sands. Med J Armed Forces

India. 2016; 
72:54-60.

2. World Health Organization, World Health Organization, Department of HIV/AIDS. Antiretroviral therapy for HIV infection in adults and adolescents: recommendations for a public health approach. 2010. Available from: http://www. ncbi.nlm.nih.gov/books/NBK138540/ (accessed Aug 28, 2018).

3. Cain LE, Phillips A, Lodi S et al. The effect of efavirenz versus nevirapine-containing regimens on immunologic, virologic and clinical outcomes in a prospective observational study. AIDS. 2012; 26:1691-1705.

4. WHO_CG_table_7.15.pdf. Available from: $h t t p: / / w w w$. who.int/hiv/pub/guidelines/arv2013/art/WHO_CG table 7.15.pdf(Accessed Sep 30, 2017)

5. Broder S. The development of antiretroviral therapy and its impact on the HIV-1/AIDS pandemic. Antiviral Res. 2010; 85:1-18.

6. Cihlar T, Ray AS. Nucleoside and nucleotide HIV reverse transcriptase inhibitors: 25 years after zidovudine. Antiviral Res. 2010; 85:39-58.

7. Adultandadolescentgl.pdf. Available from: https://aidsinfo. nih.gov/contentfiles/lvguidelines/adultandadolescentgl.pdf (Accessed Sep 30, 2017)

8. Zhou J, Phanupak P, Kiertiburanakul S, Ditangco R, Kamarulzaman A, Pujary S; TREAT Asia HIV Observational Database. Highly active antiretroviral treatment containing efavirenz or nevirapine and related toxicity in the TREAT Asia HIV Observational Database. J Acquir Immune Defic Syndr. 2006; 43:501-503.

9. Yu I, Greenberg RN, Crawford TN, Thornton AC, Myint T. Persistence of macrocytosis after discontinuation of zidovudine in HIV-infected patients. J Int Assoc Provid AIDS Care. 2017; 16:512-515.

10. Mbuagbaw L, Mursleen S, Irlam JH, Spaulding AB, Rutherford GW, Siegfried N. Efavirenz or nevirapine in three-drug combination therapy with two nucleoside or nucleotide-reverse transcriptase inhibitors for initial treatment of HIV infection in antiretroviralnaïve individuals. Cochrane Database Syst Rev. 2016; 12:CD004246.

11. Sinha S, Gupta K, Tripathy S, Dhooria S, Ranjan S, Pandey RM. Nevirapine-versus efavirenz-based antiretroviral therapy regimens in antiretroviral-naïve patients with HIV and tuberculosis infections in India: a multi-centre study. BMC Infect Dis. 2017; 17:761.

12. Kumarasamy N, Solomon S, Chaguturu SK, Mahajan AP, Flanigan TP, Balakrishnan P, Mayer KH. The safety, tolerability and effectiveness of generic antiretroviral drug regimens for HIV-infected patients in south India. AIDS. 2003; 17:2267-2269.

13. Pujari SN, Patel AK, Naik E, Patel KK, Dravid A, Patel JK, Mane AA, Bhagat S. Effectiveness of generic fixeddose combinations of highly active antiretroviral therapy for treatment of HIV infection in India. J Acquir Immune Defic Syndr. 2004; 37:1566-1569.

14. George C, Yesoda A, Jayakumar B, Lal L. A prospective study evaluating clinical outcomes and costs of three NNRTI-based HAART regimens in Kerala, India. J Clin Pharm Ther. 2009; 34:33-40.

(Received September 9, 2018; Revised October 5, 2018; Accepted October 26, 2018) 